\title{
NOTE ON THE DEGREE OF $C^{0}$-SUFFICIENCY OF PLANE CURVES
}

\author{
ANTONIO F. COSTA
}

\begin{abstract}
Let $f$ be a germ of plane curve, we define the 8 -degree of sufficiency of $f$ to be the smallest integer $r$ such that for any germ $g$ such that $j^{(r)} f=j^{(r)} g$ then there is a set of disjoint annuli in $S^{3}$ whose boundaries consist of a component of the link of $f$ and a component of the link of $g$. We establish a formula for the $\delta$-degree of sufficiency in terms of link invariants of plane curves singularities and, as a consequence of this formula, we obtain that the 8 -degree of sufficiency is equal to the $C^{0}$-degree of sufficiency
\end{abstract}

\section{0 . Introduction}

Let $f:\left(\mathrm{C}^{2}, 0\right) \rightarrow(\mathrm{C}, 0)$ be a germ of a plane curve. Given $\varepsilon>0$, we shall let $S_{\varepsilon}$ denote the sphere of radius $\varepsilon$ centered at the origin of $\mathrm{C}^{2} . \mathrm{By}[\mathrm{M}]$, there is $\eta>0$ such that for each $0<\varepsilon<\eta,\left(S_{\varepsilon}, S_{\varepsilon} \cap\{f=0\}\right)$ is a link ambient isotopic to $\left(S_{\eta}, S_{\eta} \cap\{f=0\}\right)$. We shall call the ambient isotopy class of the link $\left(S_{\eta}, S_{\eta} \cap\{f=0\}\right)$ the link of $f$ and write it $L_{f}$.

If $r$ is an integer, we let $j^{(r)} f$ denote the $r$-jet determined by $f$. There is a classical invariant of the germ $f$ that is called the " $C^{0}$-degree of sufficiency of $f^{\prime \prime}$. The definition of this invariant is the following: the integer $r$ is the $C^{0}$ degree of sufficiency of $f$ if $r$ is the smallest integer that satisfies the condition: for any germ $g$ such that $j^{(r)} f=j^{(r)} g$, then $L_{f}=L_{g}$.

The usual definition of degree of $C^{0}$-sufficiency of $f$ is given in terms of the topological type of the germ of $f$ at 0 . Remark that the link $\left(S_{\eta}, S_{\eta} \cap\{f=0\}\right)$ is ambient isotopic to the link $\left(S_{\varepsilon}, S_{\varepsilon} \cap\{g=0\}\right)$ if and only if there is an orientation preserving homeomorphism $h: S_{\eta} \rightarrow S_{\varepsilon}$ which carries $S_{\eta} \cap\{f=0\}$ to $S_{\varepsilon} \cap\{g=0\}$ (see for example [B-Z]). Applying the above result and the ones of [M], one can prove that $L_{f}=L_{g}$ if and only if $f$ and $g$ are topologically equivalent germs. Then the definition of $C^{0}$-degree of sufficiency that we give and the usual one are equivalent.

Suppose that $r$ is the degree of sufficiency of $f$, and that $g$ is a germ such that $j^{(r)} f=j^{(r)} g$. Let $\eta>0$ be a real number such that if $0<\varepsilon<\eta$, then $\left(S_{\varepsilon}, S_{\varepsilon} \cap\{f=0\} \cup S_{\varepsilon} \cap\{g=0\}\right)$ is a link of constant topological type. By the definition of the $C^{0}$-degree of sufficiency we can say that in $S_{\varepsilon}, 0<\varepsilon<\eta$, 
$S_{\varepsilon} \cap\{f=0\}$ and $S_{\varepsilon} \cap\{g=0\}$ have the same topological type, but what can we say about the relative position of the two links,i.e., which is the topological type of the link $S_{\varepsilon} \cap\{f g=0\}$ ? and how is $S_{\varepsilon} \cap\{f=0\}$ linked with $S_{\varepsilon} \cap\{g=0\}$ ?.

The purpose of this paper is to describe this relative position of the two links. By one of our results (i. e. corollary 1.2), there is a set of disjoint annuli in $S^{3}$ whose boundaries consist of a component of the link of $f$ and a component of the link of $g$.

Two germs whose links are as above will be called $\partial$-equivalents. Accordingly we shall define the $\partial$-degree of sufficiency of $f$ to be the smallest integer $r$ such that: for any germ $g$ such that $j^{(r)} f=j^{(r)} g$, then $f$ is $\partial$-equivalent to $g$.

In this paper we establish a formula for the $\partial$-degree of sufficiency in terms of link invariants of plane curves. This formula takes the same values as the formula for the $\mathrm{C}^{0}$-degree of sufficiency obtained in $[\mathrm{K}-\mathrm{L}$ ] (see also $[\mathrm{T}]$ and [Li]). Consequentily we have the equality:

$$
\partial \text {-degree of sufficiency }=C^{0} \text {-degree of sufficiency. }
$$

The author would like to thank Professor $\mathrm{C}$. Weber for helpful conversations.

\section{Definitions and results}

Let $f, g:\left(C^{2}, 0\right) \rightarrow(C, 0)$ be two germs of plane curves. We will say that " $f$ is $\partial$-equivalent to $g^{\prime \prime}$ if the link of $f$ is isotopic by disjoint annuli to the link of $g$. More precisely, let $S_{\varepsilon}$ be the sphere with center the origin and radius $\varepsilon$. The above condition means that there is an $\eta>0$, such that for every $0<\varepsilon<\eta$ there is a set of disjoint annuli $S \subset S_{\varepsilon}$ such that for each annulus $A \in S, \partial A$ consists of a component of $\{f=0\} \cap S_{\varepsilon}$ and a component of $\{g=0\} \cap S_{\varepsilon}$ (the orientation of $A$ does not induce the orientation of each component, see figure 1).

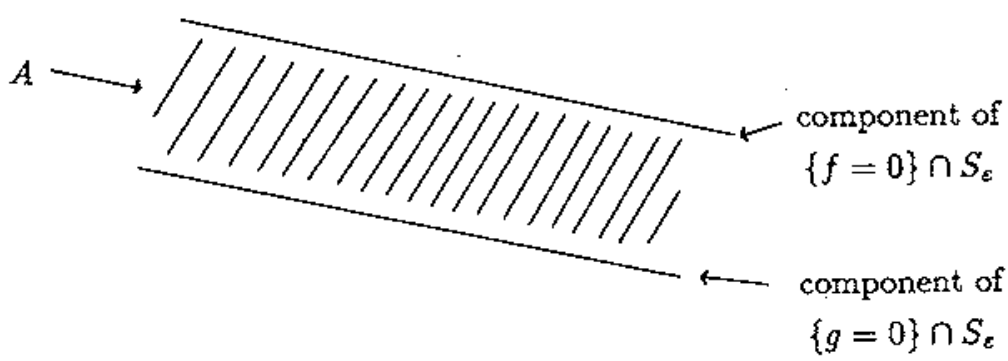

Figure 1

Example 1. Consider the germs at the origin given by the polynomials $y^{2}+x^{3}+x^{3}$ and $y^{2}+x^{3}+4 x^{7}$. They are $\partial$-equivalent and in figure 2 we show 
the annulus between the two corresponding knots.

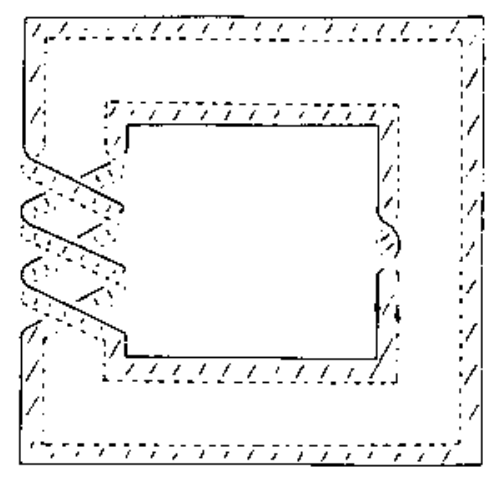

Eigure 2

Example 2. Consider now the germs given by the polynomials $y^{2}+x^{3}$ and $y^{3}+x^{2}$. They have the same topological type but they are not $\partial$-equivalent (see figure 3).

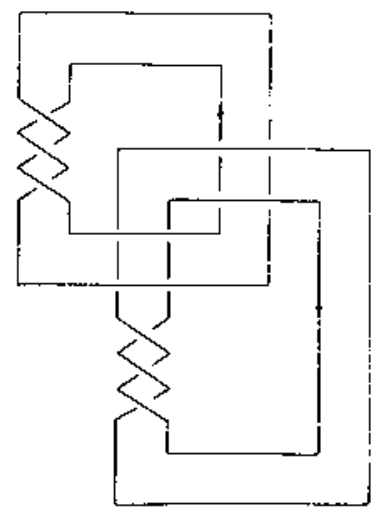

Figure 3

The link $\{f=0\} \cap S_{\varepsilon}$ is an iterated torus link. That is to say, $\{f=0\} \cap S_{\varepsilon}$ is obtained by successive satellizations of torus links (see $[M-W]$ ). Let $\left\{T_{i}\right\}$ be the minimal collection of satellization tori (unique up isotopy by the results of Jaco-Shalen and Johannson, see $[\mathbf{E}-\mathbf{N}]$ ). We split along $\left\{T_{i}\right\}$ the link exterior to obtain a finite set of picces $\left\{P_{i}\right\}$. Each of one $P_{i}$ has a Seifert fibered structure. More precisely, each piece $P_{i}$ can be considered as $S^{3}$ with a Seifert fibration with two exceptional fibers and where we have suppressed a finite number of fibers.

We denote the resolution tree of the germ $f$ by $\Gamma(f)$ and we label the strict 
transforms of the branches by an arrow ( $\uparrow$ ). If two arrows start on a same vertex then the corresponding components of the link $\{f=0\} \cap S_{\varepsilon}$ are general fbers in a same piece $P_{i}$. Then there is an annulus such that its boundary consists of the two considered components. Let $\Gamma(f g)$ be the resolution tree of the product $f g$, we label the strict transforms of the branches of $f$ with an arrow $(\uparrow)$ and the strict transforms of the branches of $g$ with a star $(\hat{W})$. If the same number of arrows and stars are attached at every vertex of $\Gamma(f g)$ then $f$ and $g$ are $\partial$-equivalent (see fig. 4).

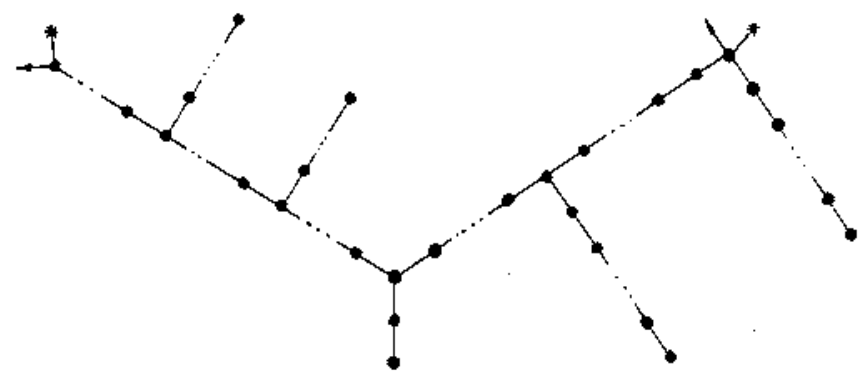

Figure 4. Exemple of $f \partial$-equivalent to $g$

Let $\varphi$ be a branch of the germ $f$. If $\Gamma(f)$ is the resolution tree of $f$ we call $v$ the vertex of $\Gamma(f)$ where the arrow of $\varphi$ start. We define $\tilde{\varphi}$ as the germ such that the resolution tree, $\Gamma(f \tilde{\varphi})$, of $f \tilde{\varphi}$ is obtained from $\Gamma(f)$ by adding a star at the vertex $v$ (see fig. 5).
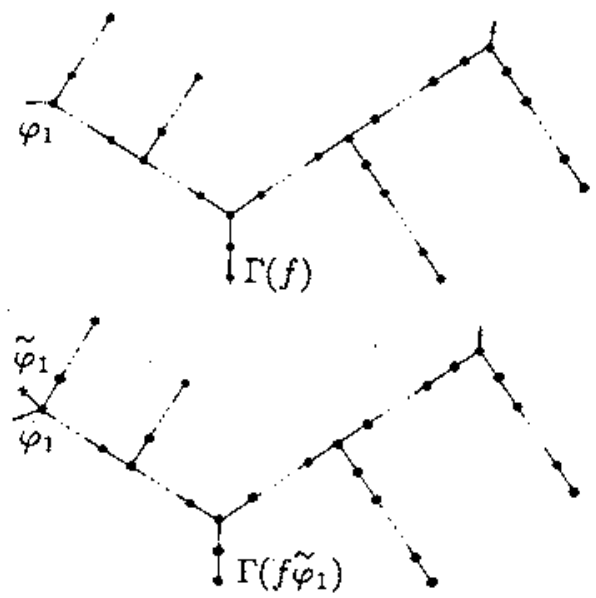

Figure 5 
In other terms, assume that the Puiseux expansion of $\varphi$ is the following:

$$
\begin{aligned}
& \sum_{i=1}^{k_{1}} a_{1 i} t^{i}+b_{1} t^{q_{1} / p_{3}}+\sum_{i=1}^{k_{2}} \alpha_{2 i} t^{\left(q_{1}+i\right) / p_{1}}+b_{2} t^{q_{2} / p_{1} p_{2}}+\ldots \\
& \ldots+b_{g-1} t^{q_{g-1} / p_{1} p_{2} \ldots p_{q-1}}+\sum_{i=0}^{\infty} c_{i} t^{\left(q_{\theta}+i\right) / p_{1} \ldots p_{g}}
\end{aligned}
$$

Replacing $c_{j}$ by $c_{j}+\varepsilon, \varepsilon>0$, we get another branch which we denote by $\varphi(j, \varepsilon)$. Then $\tilde{\varphi}$ will be the branch $\varphi(k, \varepsilon)$ where $k \geq 0$ is the minimal integer such that there exists $\varepsilon$ such that we have $I\left(\varphi(k, \varepsilon), \varphi^{\prime}\right)=I\left(\varphi, \varphi^{\prime}\right)$ for every branch $\varphi^{\prime}$ of $f$, where I denotes the intersection number.

In view of a topological interpretation of $\tilde{\varphi}$, let us consider the iterated torus link $L=\{f=0\} \cap S_{\varepsilon}$ and let $N=\{\varphi=0\} \cap S_{\varepsilon}$ be one of its components. Then the link $\{f \tilde{\varphi}=0\} \cap S_{\varepsilon}$ is obtained from $L$ by adding a general fiber of the piece which contains $N$ in the Jaco-Shalen-Johannson splitting (cf. [E-N] and $[\mathbf{M}-\mathbf{W}])$. For an easy example see fig. 6.

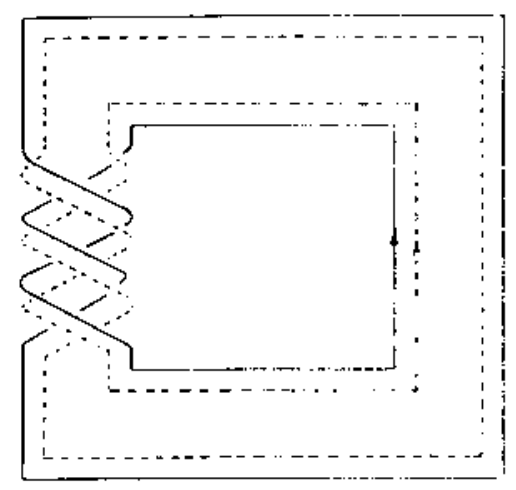

Figure 6

In this paper we establish a formula relating the $\partial$-degree of sufficiency of $f$ with the intersection numbers of the $\tilde{\varphi}$ with $f$.

Theorem 1.1. Let $f:\left(\mathrm{C}^{2}, 0\right) \rightarrow(\mathrm{C}, 0)$ be a germ of plane curve with bran. ches $\varphi_{i}, i \in I$. Let $m_{i}$ be the multiplicity of the branch $\varphi_{i}$. Then the degree of $\partial$-sufficiency $r$ is equal to:

$$
r=\max \left[\frac{I}{m_{i}} I\left(f, \widetilde{\varphi}_{i}\right)\right] .
$$

In other words $r$ is equal to the integral part of the largest polar qtotient of $f$ in the sense of Lè [L $\hat{\mathrm{e}}$ ].

The proof will be given in section 2 .

If we apply the resuit of $[\mathrm{K}-\mathrm{L}]$, then we obtain 
Corollary 1.2. Let $f:\left(\mathbb{C}^{2}, 0\right) \rightarrow(C, 0)$ be a germ of plane curve. Then: $\partial$-degree of sufficiency of $f=C^{\circ}$-degree of sufficiency of $f$.

\section{Proof of the theorem 1.1 .}

Let $f:\left(\mathrm{C}^{2}, 0\right) \rightarrow(\mathrm{C}, 0)$ be a germ of plane curve with branches $\varphi_{i}, i \in I$, and let $m_{i}, i \in I$, be the corresponding multiplicities.

We let:

$$
r=\max \left[\frac{1}{m_{i}} I\left(f, \varphi_{i}\right)\right]
$$

and

$$
s=\partial \text {-degree of sufficiency of } f \text {. }
$$

By a rotation in $\mathrm{C}^{2}$ (that does not modify $s$ ) we may suppose that the tangent cone of $f$ does not contain $x=0$.

1. Proof that $s \leq r$.

By definition of $s$ there is a germ $g$ of plane curve such that $j^{(s-1)} f=j^{(s-1)} g$ and $f$ is not $\partial$-equivalent to $g$, therefore $j^{s} f \neq j^{s} g$.

If the multiplicity of $f$ is different from the multiplicity of $g$ then $s$ is equal to the multiplicity of $f$ and hence $f$ is $\partial$-equivalent to his tangent cone. Thus $I\left(f, \tilde{\varphi}_{i}\right)=\sum_{j \in I} m_{i} m_{j}$, and

$$
r=\max _{i \in I}\left[\frac{1}{m_{i}} \sum_{j \in I} m_{i} m_{j}\right]=\sum_{j \in I} m_{j}=\text { multiplicity of } f=s .
$$

Hence we may assume that the multiplicity of $f$ is equal to the multiplicity of $g$.

Claim. If $I\left(g, \tilde{\varphi}_{i}\right)=I\left(f, \tilde{\varphi}_{i}\right)$ for every $i \in I$, there is a germ of irreducible curve $\psi$ such that for some $i \in I$ :

a.- The multiplicity of $\psi$ is equal to $m_{i}$.

b. $I\left(g, \tilde{\varphi}_{i}\right)=I(g, \psi)<I(f, \psi)$.

Proof: Consider the resolution tree, $\Gamma(f g)$, of $f g$. We label the branches of $f$ with an arrow ( $)$ and the branches of $g$ with a star $(1)$. As $f$ is not $\partial-$ equivalent to $g$ there is some vertex of $\Gamma(f g)$ where the number of arrows and stars is different. Moreover, as $f$ and $g$ have the same multiplicity, there is a vertex $P$ in $\Gamma(f g)$ where there are more arrows than stars. Let $\varphi_{i}$ be a branch corresponding to an arrow starting from $P$. If we consider now the resolution tree of $f g \tilde{\varphi}_{i}$, the arrows corresponding to $\varphi_{i}$ and $\tilde{\varphi}_{i}$ start from the same vertex of $\Gamma\left(f g \tilde{\varphi}_{i}\right)$ (see figure 7$)$. This is a consequence of the choice of $P$. We choose $\psi$ to be a branch such that $\varphi_{i}$ and $\psi$ have a new common point in $\Gamma(f g \psi)$ (see figure 8). Then it is clear that $I(g, \psi)=I\left(g, \tilde{\varphi}_{i}\right)$ and $I(f, \psi)=I\left(f, \tilde{\varphi}_{i}\right)+1$. 


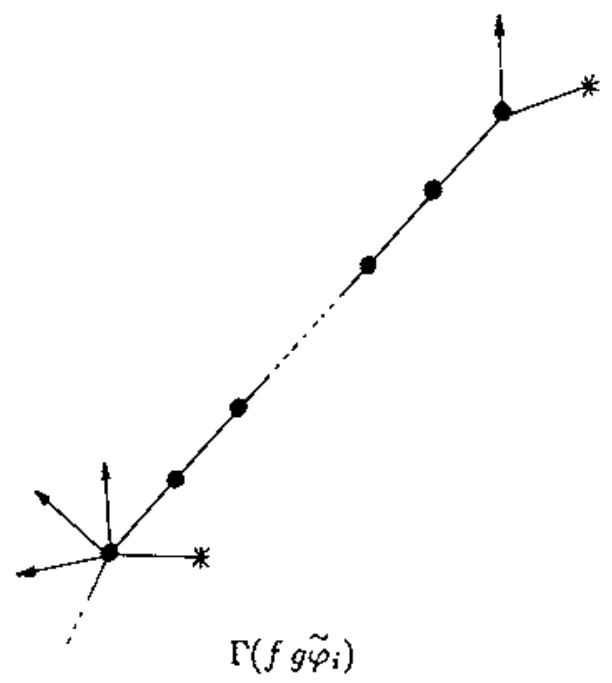

Figure 7

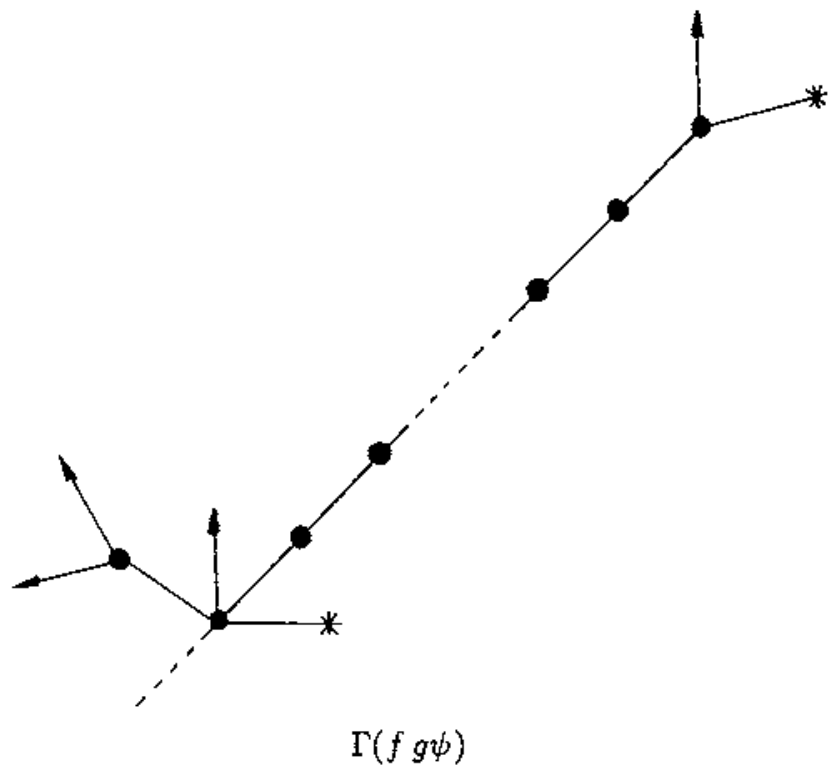

Figure 8 
We shall argue case by case.

1st. case: there is $i \in I$, such that $I\left(g, \tilde{\varphi}_{i}\right)>I\left(f, \tilde{\varphi}_{i}\right)$.

$$
\text { Let }\left\{\begin{array}{l}
X=t^{m_{i}} \\
Y=\tilde{\beta}_{i}(t)
\end{array} \text { be a parametrization of } \tilde{\varphi}_{i}\right. \text {. }
$$

Then we have: order $f\left(t^{m_{i}}, \tilde{\beta}_{i}(t)\right)<$ order $g\left(t^{m_{i}}, \tilde{\beta}_{i}(t)\right)$.

There is a term, $T(X, Y)$, of degree at least $s$ in $f(X, Y)$ (or in $g(X, Y)$ ) such that $T\left(t^{m_{i}}, \tilde{\beta}_{i}(t)\right)$ has a term of degree equal to the order of $f\left(t^{m_{i}}, \tilde{\beta}_{i}(t)\right)$. Set $T(X, Y)=X^{\alpha} Y^{\beta}$ with $\alpha+\beta \geq s$.

Then

$I\left(f, \tilde{\varphi}_{i}\right)=\operatorname{order} f\left(t^{m_{i}}, \tilde{\beta}_{i}(t)\right) \geq \alpha m_{i}+\beta\left(\operatorname{order} \tilde{\beta}_{i}(t)\right) \geq(\alpha+\beta) m_{i} \geq s m_{i}$.

That is:

$$
s \leq\left[\frac{\left(f, \tilde{\varphi}_{i}\right)}{m_{i}}\right] \leq T .
$$

2nd case. $I\left(g, \tilde{\varphi}_{i}\right)<I\left(f, \tilde{\varphi}_{i}\right)$ for some $i \in I$.

Then the order of $f\left(t^{m_{i}}, \tilde{\beta}_{i}(t)\right)$ is greater than the order of $g\left(t^{m_{i}}, \tilde{\beta}_{i}(t)\right)$. There is a term, $T(X, Y)$, of degree at least $s$ in $f(X, Y)$ or $g(X, Y)$ such that $T\left(t^{m_{i}}, \tilde{\beta}_{i}(t)\right)$ has a term of degree equal to the order of $g\left(t^{m_{i}}, \tilde{\beta}_{i}(t)\right)$. Then:

$$
s \leq\left[\frac{I\left(f, \tilde{\varphi}_{i}\right)}{m_{i}}\right]<\left[\frac{I\left(f, \tilde{\varphi}_{i}\right)}{m_{i}}\right] \leq r
$$

3rd case. $I\left(f, \tilde{\varphi}_{i}\right)=I\left(g, \tilde{\varphi}_{i}\right)$ for every $i \in I$.

Let $\psi$ be the branch given by the claim. By construction the multiplicity of $\psi$ is equal to $m_{i}$ for some $i \in I$.

$$
\text { Let }\left\{\begin{array}{l}
X=t^{m_{i}} \\
Y=\tilde{\beta}(t)
\end{array} \text { be a parametrization of } \psi\right.
$$

A.s $I(g, \psi) \therefore I(f, \psi)$ we have:

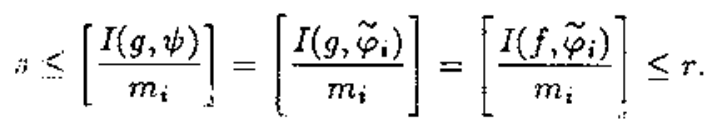




\section{Proof that $r \leq s$.}

By the remark that we made in the introduction this inequality is a consequence of the result of Kuo and Lu. Here is an easy direct argument:

Assume that $s<r$. Then, if $j^{(r-1)} f=j^{(r-1)} g$, we have that $f$ and $g$ are $\partial$-equivalent, and let $S$ be a set of disjoint annuli verifying the conditions of the definition of $\partial$-equivalence. If $\varphi_{i}$ is a branch of $f$ we may choose $\tilde{\varphi}_{i}$ in such a way that the knot corresponding to $\tilde{\varphi}_{i}, \tilde{N}_{i}$, does not cut the annuli of $S$. Then the linking number of $\tilde{N}_{i}$ with each component $N_{j}$ of the link of $f$, is equal to the linking number of the component of the link $g$ in the annulus which contains $N_{j}$. Then $I\left(f, \tilde{\varphi}_{i}\right)=I\left(g, \tilde{\varphi}_{i}\right)$.

In particular, if we call $f_{r-1}$ the set of terms of degree at most $r-1$ in $f$, we have:

$I\left(f_{r-1}, \tilde{\varphi}_{i}\right)=I\left(f_{r-1}+a X^{r}, \tilde{\varphi}_{i}\right)$, for all $a \in C-\{0\}$, and for every $i \in I$.

$$
\text { Let }\left\{\begin{array}{c}
X=t^{m_{i}} \\
Y=\tilde{\beta}(t)
\end{array} \text { be a parametrization of } \tilde{\varphi}_{i} .\right.
$$

Then: order $f_{r-1}\left(t^{m_{i}}, \tilde{\beta}(t)\right)=\operatorname{order}\left(f_{r-1}\left(t^{m_{i}}, \tilde{\beta}(t)\right)\right.$ at $\left.{ }^{r m_{i}}\right)$ for all $a \in \mathrm{C}-$ $\{0\}$. This implies that order $f_{r-1}\left(t^{m_{i}}, \tilde{\beta}(t)\right)<r m_{i}$ for every $i \in Y$.

Then $r=\max \left[\frac{I\left(f, \tilde{\varphi}_{i}\right)}{m_{i}}\right]=\max \left[\frac{I\left(f_{r-1}, \tilde{\varphi}_{i}\right)}{m_{i}}\right]<r$, which is a contradiction.

\section{References}

[B-Z] Burde G. AND Zieschang H., Knots, Berlin, New York: de Gruyter (1985).

[E-N] EISENBUD D. AND NEUMANN W., Three-dimensional link theory and invariants of plane curve singularities, Ann. of Math. Studies 110 (1985), Univ. Press, Princeton, N. J..

[K-L] KUO T.C. AND LU Y.C., On Analytic function germs of two complex variables, Topology 16 (1977), 299-310.

[Lê] LÂE D ŨNG TRÁNG, Courbes polaires et résolution des courbes planes, Géométrie algébrique et applications II, Trabaux en Cours 23 (1987), Paris: Hermann.

[Li] LICHTIN B., Estimates and formulae for the $C^{\circ}$-degree of sufficiency of plane curves, Proc. Simp. in Pure Maths 40 Part 2 (1983), 155-160.

[M] MiLnor J., Singularities of complex hypersurfaces, Ann. of Math. Studies 61 (1968), Princeton Univ. Press, Princeton N.J.. 
[M-W] MicheL F. AND WEBER C., Topologie des germes de courbes planes à plusieurs branches, Preprint (1985).

[T] TEISSIER B., Varietés polaires I. Invariants polaires des singularités d'hypersurfaces, Inv. Math. 40 (1977), 267-292.

\author{
Dpto. de Matemáticas Fundamentales \\ Facultad de Ciencias, U.N.E.D. \\ 28040 Madrid \\ SPAIN
}

Rebut el 15 de gener de 1988 